\title{
Sexuality during COVID lockdown: a cross-sectional Italian study among hospital workers and their relatives
}

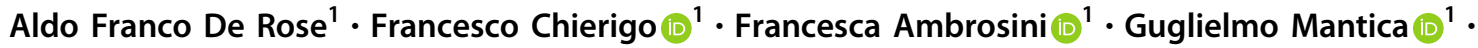 \\ Marco Borghesi ${ }^{1,2} \cdot$ Nazareno Suardi ${ }^{1,2} \cdot$ Carlo Terrone $^{1,2}$
}

Received: 21 June 2020 / Revised: 15 November 2020 / Accepted: 1 December 2020 / Published online: 5 January 2021

(c) The Author(s), under exclusive licence to Springer Nature Limited part of Springer Nature 2021

\begin{abstract}
In March 2020, the Italian Government introduced measures to reduce the spread of COVID-19 infection. Between 8th April and 2nd May 2020 we investigated levels and correlates of sexual activity and depression during COVID-19 lockdown in a sample of hospital workers and their acquaintances by an online survey on SurveyMonkey. Socio-demographic data, International Index of Erectile Function, Female Sexual Function Index, and Beck Depression Inventory were recorded. Multivariable logistic regression analysis (MLRA) was used to test predictors of depressive symptoms and low sexual desire and satisfaction. A statistically significant difference in age, change in working habit, sexual satisfaction, sexual desire, and depressive symptoms was found between males and females. A statistically significant higher proportion of health care workers had low sexual desire $(65.3 \%$ vs $56.8 \%, p=0.042)$. At MLRA, age, being female, being a health care worker, having children at home, living with the partner, and having low sexual satisfaction were predictors of low level of sexual desire. To our knowledge, this is one of the few studies using validated questionnaires for both males and females to assess sexual well-being and psychometric alterations during COVID quarantine.
\end{abstract}

\section{Introduction}

Since late December 2019, a novel coronavirus (COVID19) was identified as the cause of a cluster of pneumonia cases in the city of Wuhan, in the Hubei Province, China [1]. It has rapidly spread throughout China, followed by an increasing number of cases world widely.

SARS-CoV-2 spreads by direct and contact transmission [2]. Most patients only suffer from mild symptoms or are asymptomatic, but almost one out of five experiences severe disease needing hospitalization and oxygen support, and 5\% may require an intensive care unit admission. In severe

These authors contributed equally: De Rose Aldo Franco, Chierigo Francesco

Francesco Chierigo

francesco.chierigo@gmail.com

1 Department of Urology, Policlinico San Martino Hospital, University of Genova, Genova, Italy

2 Department of Surgical and Diagnostic Integrated Sciences (DISC), University of Genova, Genova, Italy cases, SARS-CoV-2 infection may lead to acute respiratory distress syndrome [3].

On March 11th 2020, the World Health Organization declared the COVID-19 outbreak a global pandemic. Since then, the primary goal has been to slow down the spread of the infection: mobility and social restrictions have been imposed in numerous countries around the world, and all nonessential businesses have been closed (COVID-19 shutdown or lockdown). Most political, cultural, religious, and sporting events have been cancelled or postponed. By Spring 2020, almost half of the world population was in lockdown [4]. Italy also had to face a lockdown from March to May 2020.

Life during the pandemic can be characterized by several stress factors deriving from domestic isolation, limited physical and social activities, economic uncertainties, and fear of contagion and death. These stressors can negatively impact the psychological well-being of individuals, by triggering anxiety and depression [5]. Clinical depression may lead to changes in sexual behaviour, such as a reduction in sexual interest and sexual response, which may be more marked in women than in men [6]. Issues of sexual and reproductive health and sexual well-being are closely related to overall health and quality of life and should not be 
forgotten even (and perhaps especially) in such times of crisis.

Hospital workers present several peculiarities: most of them has maintained a daily routine, but may have experienced an increase in workload, new task and responsibilities and, being in close contact with COVID-19 patients, may have been at higher risk of contagion. Therefore, their emotional and sexual spheres may have been affected differently than those of the general population.

In this study, we aimed to investigate the effect of COVID-19 lockdown on sexuality and depressive symptoms among hospital workers and their relatives and friends in Italy.

\section{Materials and methods}

During Italian lockdown, from 8th April to 2nd May, we conducted a voluntary online anonymous survey on SurveyMonkey among a convenience sample selected as follows: we invited heath care workers of our institution to fill in the questionnaires and invited them to share the survey link with their relatives and friends. The link of the survey was mainly shared by WhatsApp, Facebook, Telegram, and e-mail. Duplicate entries were avoided by preventing users with the same IP address access to the survey twice by the specific options on SurveyMonkey. This study was approved by our Institutional Review Board (Osp. Pol. S. Martino IRCCS, 473/2020). All participants provided informed consent before filling in the questionnaire. No incentives were offered. Socio-demographic data recorded included sex, age, and educational level. We asked responders whether they were living with their sexual partners during lockdown, whether they had children at home during lockdown and if their working habit changed as a consequence of the lockdown. Participants filled out questionnaires about their sexual habits: males completed the International Index of Erectile Function (IIEF) [7] in its Italian 15-items version and females completed the Italian version of the Female Sexual Function Index (FSFI) [8, 9]. Adaptive questioning was used to make males and females fill in only IIEF-15 and FSFI, respectively. To objectively assess levels of sexual satisfaction, we measured the IIEF15 overall satisfaction (OS) and intercourse satisfaction (IS) domains for males and the FSFI satisfaction (Sat) domains for females. We defined low satisfaction levels using an arbitrary cut-off score $\leq 12.5$ in the sum of IIEF-IS and IIEFOS domains for males, and an arbitrary cut-off score $\leq 7.5$ in the FSFI Satisfaction domain for females. Sexual desire (SD) domain of IIEF and FSFI were also recorded and we defined low sexual desire using an arbitrary cut-off score $\leq 5$ for both IIEF-SD and FSFI-SD domains. The Italian validated version of the Beck Depression Inventory (BDI)
[10, 11] was used to assess the presence of depressive symptoms. Depressive symptoms were defined as absent or minor for BDI scores from 0 to 13 , mild from 14 to 19 , moderate from 20 to 28 and severe from 29 to 63 [12]. Mann-Whitney and chi-squared tests were used to assess the difference between age, level of education, working habit, children at home, living with partner, depressive symptoms, and sexual desire and satisfaction. Multivariable logistic regression analysis was used to test possible predictors of depression, defined as BDI score $\geq 13$. The same analysis was used to test possible predictors of abnormal sexual desire and satisfaction. Statistical analyses were performed using SPSS v.24 (IBM Corp., Armonk, NY, USA).

\section{Results}

Table 1 lists clinical and socio-demographic characteristics for the whole cohort of responders. Overall, $260(47.8 \%)$ males and $284(52.2 \%)$ females responded to the online survey. Median (IQR) age was 31 (27-45). Median (IQR) IIEF-15 was 25 (17-29) and median (IQR) IIEF-OS, IIEF-IS and IIEF-SD were 5 (4-9), 5 (0-7) and 4 (3-5), respectively. Median (IQR) FSFI was 16 (5-19), while the median (IQR) for FSFI-Sat and FSFI was 4 (0-6). As shown in Table 2, a statistically significant difference in age, change in working habit, sexual satisfaction, sexual desire, and depressive symptoms was found between males and females (all $p<$ $0.05)$. We also performed a subgroup analysis comparing health care workers $(n=294,54 \%)$ with other responders $(n=250,46 \%)$, as shown in Table 3 . Of note, health care workers responding to the questionnaire were younger, with a higher level of education, were living without their partner and had no children at home, and saw no difference in working habit in comparison with other responders. We found a statistically significant difference in low sexual desire $(65.3 \%$ health care workers vs $56.8 \%$ other responders, $p=0.042$ ), but no difference was found in satisfaction, with $91.2 \%$ of health care workers and $87.2 \%$ of other responders reporting low satisfaction. As far as depressive symptoms are concerned, health care workers showed a statistically significant higher BDI score, even if no difference was found when BDI scores were divided in subcategories.

At multivariate logistic regression analysis, only age [OR $=0.947,95 \%$ CI $(0.917,0.979), p=0.001]$, medium level of education $[\mathrm{OR}=1.920,95 \%$ CI $(1.030,3.578)$, $p=0.040]$ and sexual satisfaction $[\mathrm{OR}=0.386,95 \% \mathrm{CI}$ (0.177, 0.845), $p=0.017]$ were found to be independent predictors of the presence of depression (Table 4).

At the same analysis, age $[\mathrm{OR}=0.948,95 \%$ CI $(0.929$, $0.969), p<0.001]$, being female $[\mathrm{OR}=0.070,95 \% \mathrm{CI}$ 
Table 1 Socio-demographic and psychometric characteristics of the entire population.

\begin{tabular}{|c|c|}
\hline \multicolumn{2}{|l|}{ Gender, N (\%) } \\
\hline Male & $260(47.8 \%)$ \\
\hline Female & $284(52.2 \%)$ \\
\hline Age, years, Median (IQR) & $31(27,45)$ \\
\hline \multicolumn{2}{|l|}{ Level of education, $\mathrm{N}(\%)$} \\
\hline High & $270(49.6 \%)$ \\
\hline Medium & $240(44,1 \%)$ \\
\hline Low & $34(6.3 \%)$ \\
\hline \multicolumn{2}{|l|}{ Working habit, $\mathrm{N}(\%)$} \\
\hline Normal & $264(48.5 \%)$ \\
\hline Smart-working & $160(29.4 \%)$ \\
\hline Interruption & $120(22.1 \%)$ \\
\hline \multicolumn{2}{|l|}{ Living with partner, $\mathrm{N}(\%)$} \\
\hline Yes & $286(52.6 \%)$ \\
\hline No & $258(47.4 \%)$ \\
\hline \multicolumn{2}{|l|}{ Children at home, $\mathrm{N}(\%)$} \\
\hline Yes & $156(28.7 \%)$ \\
\hline No & $388(71.3 \%)$ \\
\hline IIEF-Erectile Function, Median (IQR) & $10(3,11)$ \\
\hline IIEF-Orgasmic Function, Median (IQR) & $3(3,5)$ \\
\hline IIEF-Sexual Desire, Median (IQR) & $4(3,5)$ \\
\hline IIEF-Intercourse Satisfaction, Median (IQR) & $5(0,7)$ \\
\hline IIEF-Overall Satisfaction, Median (IQR) & $5(4,9)$ \\
\hline IIEF-Total, Median (IQR) & $25(17,29)$ \\
\hline FSFI-Desire, Median (IQR) & $6(4,7)$ \\
\hline FSFI-Arousal, Median (IQR) & $4(0,6)$ \\
\hline FSFI-Lubrification, Median (IQR) & $12(0,13)$ \\
\hline FSFI-Orgasm, Median (IQR) & $7(0,8)$ \\
\hline FSFI-Satisfaction, Median (IQR) & $4(0,6)$ \\
\hline FSFI-Pain, Median (IQR) & $10(0,15)$ \\
\hline FSFI-Overall, Median (IQR) & $16(5,19)$ \\
\hline BDI-Cognitive, Median (IQR) & $2(0,4)$ \\
\hline BDI-Somatic/Affective, Median (IQR) & $4(1,7)$ \\
\hline BDI-Tot, Median (IQR) & $7(2,12)$ \\
\hline \multicolumn{2}{|l|}{ Depressive symptoms, N (\%) } \\
\hline No/Minimal & $472(86.8 \%)$ \\
\hline Mild & $36(6.6 \%)$ \\
\hline Moderate & $26(4.8 \%)$ \\
\hline Severe & $10(1.8 \%)$ \\
\hline
\end{tabular}

(0.041, 0.120), $p<0.001]$, being a health care worker $[\mathrm{OR}=2.632,95 \% \mathrm{CI}(1.463,4.737), p=0.001]$, having children at home $[\mathrm{OR}=0.555,95 \% \mathrm{CI}(0.346,0.889), p=$ $0.014]$, living with the partner [OR $=0.636,95 \% \mathrm{CI}(0.410$, $0.985), p=0.043$ ], and having low sexual satisfaction $[\mathrm{OR}=3.800,95 \% \mathrm{CI}(1.835,7.872), \mathrm{p}<0.001]$ were predictors of low level of sexual desire (Table 5).
Table 2 Comparison of socio-demographic and psychometric characteristics between males and females.

\begin{tabular}{|c|c|c|c|}
\hline & $\begin{array}{l}\text { Male, } n=260 \\
(47.8 \%)\end{array}$ & $\begin{array}{l}\text { Female, } \\
n=284 \\
(52.2 \%)\end{array}$ & $p$ value \\
\hline $\begin{array}{l}\text { Age, years, } \\
\text { Median (IQR) }\end{array}$ & $33(28,49)$ & $30(27,42)$ & 0.002 \\
\hline Level of education, $\mathrm{N}(\%)$ & & & 0.2 \\
\hline High & $135(51.9 \%)$ & $135(47.5 \%)$ & \\
\hline Medium & $106(40.8 \%)$ & $134(47.2 \%)$ & \\
\hline Low & $19(7.3 \%)$ & $15(5.3 \%)$ & \\
\hline Living with partner, $\mathrm{N}(\%)$ & & & 0.4 \\
\hline Yes & $142(54.6 \%)$ & $144(50.7 \%)$ & \\
\hline No & $118(45.4 \%)$ & $140(49.3 \%)$ & \\
\hline Children at home, $\mathrm{N}(\%)$ & & & 0.6 \\
\hline Yes & $72(27.7 \%)$ & $84(29.6 \%)$ & \\
\hline No & $188(72.3 \%)$ & $200(70.4 \%)$ & \\
\hline Working habit, $\mathrm{N}(\%)$ & & & 0.004 \\
\hline Normal & $112(43.1 \%)$ & $152(53.5 \%)$ & \\
\hline Smart-working & $94(36.2 \%)$ & $66(23.2 \%)$ & \\
\hline Interruption & $54(20.8 \%)$ & $56(23.2 \%)$ & \\
\hline Sexual desire ${ }^{\mathrm{a}}$ & & & $<0.001$ \\
\hline Low & $214(82.3 \%)$ & $120(42.3 \%)$ & \\
\hline Normal & $46(17.7 \%)$ & $164(57.7 \%)$ & \\
\hline Satisfaction $^{\mathrm{b}}$ & & & 0.032 \\
\hline Low & $240(92.3 \%)$ & $246(86.6 \%)$ & \\
\hline Normal & $20(7.7 \%)$ & $38(13.4 \%)$ & \\
\hline $\begin{array}{l}\text { BDI-Cognitive, } \\
\text { Median (IQR) }\end{array}$ & $1(0,4)$ & $2(1,5)$ & $<0.001$ \\
\hline $\begin{array}{l}\text { BDI-Somatic/Affective, } \\
\text { Median (IQR) }\end{array}$ & $3(0,5)$ & $6(2,8)$ & $<0.001$ \\
\hline BDI-Tot, Median (IQR) & $5(1,10)$ & $8(4,12)$ & $<0.001$ \\
\hline $\begin{array}{l}\text { Depressive } \\
\text { symptoms, N (\%) }\end{array}$ & & & 0.016 \\
\hline No/Minimal & $236(90.8 \%)$ & $236(83.1 \%)$ & \\
\hline Mild & $10(3.8 \%)$ & $26(9.2 \%)$ & \\
\hline Moderate & $8(3.1 \%)$ & $18(6.3 \%)$ & \\
\hline Severe & $6(2.3 \%)$ & $4(1.4 \%)$ & \\
\hline
\end{tabular}

${ }^{a}$ Sexual desire was defined low for a score $\leq 5$ in the sexual desire domains of IIEF and FSFI for males and female, respectively.

${ }^{\mathrm{b}}$ Satisfaction levels were defined as a score $\leq 12.5$ in the sum of IIEF-IS and IIEF-OS domains for males, and a score $\leq 7.5$ in the FSFI Satisfaction domain for females. Mann-Witney and chi-squared tests were used when appropriate.

As far as sexual satisfaction is concerned, age [OR $=$ $0.973,95 \%$ CI $(0.948,0.998), p=0.037]$, low level of education $[\mathrm{OR}=0.231,95 \% \mathrm{CI}(0.077,0.693), p=0.009]$, living with partner $[\mathrm{OR}=0.492,95 \% \mathrm{CI}(0.256,0.945)$, $p=0.033$ ], low sexual desire [OR $=3.885,95 \% \mathrm{CI}(1.878$, 8.037), $p<0.001]$ and depression $[\mathrm{OR}=0.378,95 \% \mathrm{CI}$ (0.172, 0.829), $p=0.015]$ were found to be independent 
Table 3 Comparison of socio-demographic and psychometric characteristics between health care workers and non-health care workers.

\begin{tabular}{|c|c|c|c|}
\hline & $\begin{array}{l}\text { Health care } \\
\text { workers, } n= \\
294(54 \%)\end{array}$ & $\begin{array}{l}\text { Non health care } \\
\text { workers, } n= \\
250(46 \%)\end{array}$ & $p$ value \\
\hline Age, years, Median (IQR) & $29(27,36)$ & $36(27,49)$ & 0.006 \\
\hline Gender, N (\%) & & & $<0.001$ \\
\hline Male & $114(38.8 \%)$ & $146(58.4 \%)$ & \\
\hline Female & $180(61.2 \%)$ & $104(41.6 \%)$ & \\
\hline Level of education, $\mathrm{N}(\%)$ & & & $<0.001$ \\
\hline High & $194(66 \%)$ & $76(30.4 \%)$ & \\
\hline Medium & $100(34 \%)$ & $140(43.2 \%)$ & \\
\hline Low & $0(0 \%)$ & $34(13.6 \%)$ & \\
\hline Living with partner, $\mathrm{N}(\%)$ & & & 0.004 \\
\hline Yes & $138(46.9 \%)$ & $148(59.2 \%)$ & \\
\hline No & $156(53.1 \%)$ & $102(40.8 \%)$ & \\
\hline Children at home, $\mathrm{N}(\%)$ & & & 0.05 \\
\hline Yes & $74(25.2 \%)$ & $82(32.8 \%)$ & \\
\hline No & $220(74.8 \%)$ & $168(67.2 \%)$ & \\
\hline Working habit, N (\%) & & & $<0.001$ \\
\hline Normal & $218(74.1 \%)$ & $46(18.4 \%)$ & \\
\hline Smart-working & $42(14.3 \%)$ & $118(47.2 \%)$ & \\
\hline Interruption & $34(11.6 \%)$ & $86(34.4 \%)$ & \\
\hline Sexual desire ${ }^{a}$ & & & 0.042 \\
\hline Low & $192(65.3 \%)$ & $142(56.8 \%)$ & \\
\hline Normal & $102(34.7 \%)$ & $108(43.2 \%)$ & \\
\hline Satisfaction $^{\mathrm{b}}$ & & & 0.136 \\
\hline Low & $268(91.2 \%)$ & $218(87.2 \%)$ & \\
\hline Normal & $26(8.8 \%)$ & $32(12.8 \%)$ & \\
\hline $\begin{array}{l}\text { BDI-Cognitive, } \\
\text { Median (IQR) }\end{array}$ & $2(1,6)$ & $1(0,3)$ & $<0.001$ \\
\hline $\begin{array}{l}\text { BDI-Somatic/Affective, } \\
\text { Median (IQR) }\end{array}$ & $5(2,8)$ & $4(1,6)$ & 0.001 \\
\hline BDI-Tot, Median (IQR) & $8(3,12)$ & $6(1,10)$ & $<0.001$ \\
\hline $\begin{array}{l}\text { Depressive } \\
\text { symptoms, N (\%) }\end{array}$ & & & 0.114 \\
\hline No/Minimal & $248(84.4 \%)$ & $224(89.6 \%)$ & \\
\hline Mild & $24(8.2 \%)$ & $12(4.8 \%)$ & \\
\hline Moderate & $18(6.1 \%)$ & $8(3.2 \%)$ & \\
\hline Severe & $4(1.4 \%)$ & $6(2.4 \%)$ & \\
\hline
\end{tabular}

${ }^{a}$ Sexual desire was defined low for a score $\leq 5$ in the sexual desire domains of IIEF and FSFI for males and female, respectively.

${ }^{\mathrm{b}}$ Satisfaction levels were defined as a score $\leq 12.5$ in the sum of IIEF-IS and IIEF-OS domains for males, and a score $\leq 7.5$ in the FSFI Satisfaction domain for females. Mann-Witney and chi-squared tests were used when appropriate.

predictors at multivariate logistic regression analysis (Table 6).

\section{Discussion}

In our study, sexual desire was found to be low in more than $80 \%$ of males and more than $40 \%$ of females. Our results are confirmed by those by Schiavi et al. [13], who reported a statistically significant difference $(p=0.007)$ in FSFI desire domain between before-COVID period (mean \pm standard
Table 4 Multivariate logistic regression analysis testing possible predictors of depression ${ }^{\mathrm{a}}$.

\begin{tabular}{lll}
\hline & OR $(95 \%$ CI $)$ & $p$ value \\
\hline Age & $0.947(0.917,0.979)$ & 0.001 \\
Gender (male as reference) & $1.546(0.839,2.849)$ & 0.162 \\
$\begin{array}{l}\text { Level of education (high as reference) } \\
\quad \text { Medium }\end{array}$ & $1.920(1.030,3.578)$ & 0.040 \\
$\quad$ Low & $1.148(0.307,4.298)$ & 0.838 \\
Health care worker (no as reference) & $1.987(0.946,4.174)$ & 0.070 \\
Work habit (normal as reference) & & \\
$\quad$ Smart-working & $1.278(0.614,2.660)$ & 0.511 \\
$\quad$ Interruption & $1.714(0.823,3.572)$ & 0.150 \\
Living with partner (no as reference) & $0.880(0.511,1.515)$ & 0.643 \\
$\begin{array}{l}\text { Children at home (no as referenceI) } \\
\text { Sexual desire (normal as }\end{array}$ & $0.971(0.526,1.794)$ & 0.925 \\
referecence) & $1.213(0.645,2.281)$ & 0.549 \\
$\begin{array}{l}\text { Sexual satisfaction (normal as } \\
\text { reference) }\end{array}$ & $0.386(0.177,0.845)$ & 0.017 \\
\hline
\end{tabular}

${ }^{\mathrm{a}}$ Depression was defined as BDI score $\geq 13$.

Table 5 Multivariate logistic regression analysis testing possible predictors of low sexual desire . $^{\mathrm{a}}$

\begin{tabular}{|c|c|c|}
\hline & OR $(95 \% \mathrm{CI})$ & $p$ value \\
\hline Age & $0.948(0.929,969)$ & $<0.001$ \\
\hline Gender (male as reference) & $0.070(0.041,0.120)$ & $<0.001$ \\
\hline \multicolumn{3}{|l|}{ Level of education (high as reference) } \\
\hline Medium & $2.325(1.423,3.798)$ & 0.001 \\
\hline Low & $2.587(0.950,7.042)$ & 0.063 \\
\hline Health care worker (no as reference) & $2.632(1.463,4.737)$ & 0.001 \\
\hline \multicolumn{3}{|l|}{ Work habit (normal as reference) } \\
\hline Smart-working & $0.899(0.496,1.628)$ & 0.725 \\
\hline Interruption & $0.771(0.423,1.406)$ & 0.396 \\
\hline Living with partner (no as reference) & $0.636(0.410,0.985)$ & 0.043 \\
\hline Children at home (no as referenceI) & $0.555(0.346,0.889)$ & 0.014 \\
\hline $\begin{array}{l}\text { Sexual satisfaction (normal as } \\
\text { reference) }\end{array}$ & $3.800(1.835,7.872)$ & $<0.001$ \\
\hline Depression (no as reference) ${ }^{b}$ & $1.357(0.727,2.533)$ & 0.33 \\
\hline
\end{tabular}

${ }^{a}$ Sexual desire was defined low for a score $\leq 5$ in the sexual desire domains of IIEF and FSFI for males and female, respectively.

${ }^{\mathrm{b}}$ Depression was defined as BDI score $\geq 13$.

deviation $(\mathrm{SD})=3.8 \pm 0.9)$ and during-COVID period (mean $\pm \mathrm{SD}=3.2 \pm 1.1$ ). Although social and cultural differences may exist, a much lower proportion of deteriorated sexual desire (22\%) was found among young Chinese students during COVID-19 pandemic [14]. However, our finding is in contrast with results by Cocci et al. [15], who found out that more than $40 \%$ of responders of their online survey reported an increased sexual desire during lockdown, and by Yuksel et al [16], who found out a significantly $(p=0.011)$ higher FSFI desire domain score 
Table 6 Multivariate logistic regression analysis testing possible predictors of low sexual satisfaction ${ }^{\mathrm{a}}$.

\begin{tabular}{lll}
\hline & OR $(95 \%$ CI $)$ & $p$ value \\
\hline Age & $0.973(0.948,0.998)$ & 0.037 \\
Gender (male as reference) & $0.777(0.370,1.634)$ & 0.506 \\
Level of education (high as reference) & \\
$\quad$ Medium & $1.284(0.629,2.619)$ & 0.492 \\
$\quad$ Low & $0.231(0.077,0.693)$ & 0.009 \\
Health care worker (no as reference) & $1.065(0.474,2.393)$ & 0.878 \\
Work habit (normal as reference) & & \\
$\quad$ Smart-working & $1.110(0.469,2.627)$ & 0.812 \\
$\quad$ Interruption & $0.886(0.399,1.966)$ & 0.765 \\
Living with partner (no as reference) & $0.492(0.256,0.945)$ & 0.033 \\
Children at home (no as referenceI) & $1.086(0.558,2.114)$ & 0.809 \\
Sexual desire (normal as reference) & $3.885(1.878,8.037)$ & $<0.001$ \\
Depression (no as reference) & $0.378(0.172,0.829)$ & 0.015 \\
\hline
\end{tabular}

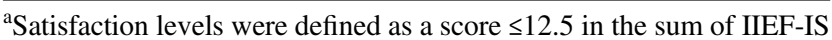
and IIEF-OS domains for males, and a score $\leq 7.5$ in the FSFI Satisfaction domain for females.

${ }^{\mathrm{b}}$ Depression was defined as BDI score $\geq 13$.

during the pandemic (mean $\pm \mathrm{SD}=3.94 \pm 1.36$ ) than before the pandemic (mean $\pm \mathrm{SD}=3.42 \pm 1.20$ ). A low sexual desire was associated with being female, being a health care worker, having children at home, living with the partner, and having low sexual satisfaction. In Italy, most household duties still fall disproportionately on women. COVID-19 emergency may have worsened this condition, by prevented women from being helped from the outside (baby-sitters, extended family, housekeepers, etc.). This increase in workload may help explain the lower sexual desire in women. In addition, low sexual desire in people living with their partner can be explained by the fear of infecting them and by the increased tensions which may arise in such periods of uncertainties. Furthermore, the constant presence of children at home due to prolonged school closure and the suspension of extracurricular activities may have reduced the possibilities of intimacy for the parents. Last, it may be that the psychological burden and the knowledge of being at increased risk of infection experienced by health care workers may have negatively impacted their sexual desire.

All studies in our knowledge found a decrease in sexual satisfaction during COVID pandemic. Cocci et al [15]. reported a significant reduction in sexual satisfaction during the quarantine, with more than $50 \%$ of the respondents describing a complete absence of sexual satisfaction. Much lower proportion of reduced sexual satisfaction $(15 \%)$ was reported by Li G. et al., with students in an exclusive relationship at the time of the pandemic being more likely to be dissatisfied with their sexual life [14]. In a study conducted on Turkish women [16], a statistically significant decrease in FSFI satisfaction domain was seen. Results were confirmed by an Italian study on reproductive-age women [13], reporting an average decrease of FSFI satisfaction domain of almost 2 points during lockdown. Similarly, we found that about $90 \%$ of our responders reported low levels of sexual satisfaction, irrespectively of gender or being health care workers. On multivariate logistic regression analysis, low levels of sexual satisfaction could be predicted by depression, low sexual desire and living with their partner. The association between depression and low sexual satisfaction is widely studied [17-21]. In our population, it is likely that sexual satisfaction was negatively influenced by the psychological distress caused by the pandemic, especially in couples living together who might have experienced a deterioration in their relationship. Responders who were living alone might have acknowledged the impossibility of having sexual intercourse and this may have had a mitigation effect on their sexual satisfaction scoring.

Unfortunately, we did not investigate whether sexual dissatisfaction and absence of sexual desire were correlated with an increase in autoerotism and use of pornography. Indeed, it is widely accepted that pornography use is associated with decreased sexual satisfaction [22] and Cocci et al [15]. reported an increase of autoerotism during lockdown in $40 \%$ of the participants of their survey. Other limitations of this study might be the reliance upon self-reporting questionnaires and the constantly changing prevalence of COVID-19, which means that our findings only provide cross-sectional data based on a local convenient sample. Another possible limitation to our study is that, to our knowledge, the Italian version of IIEF-15 is not validated. Moreover, we used arbitrary cutoff values to define low levels of sexual desire and satisfaction of both male and females. However, to our knowledge, there are no studies clearly defining a cut-off value for abnormal satisfaction domains for both IIEF-15 and FSFI, especially when dealing with the general population. In a study by Rosen et al [7], the IIEF was tested in a series of 111 men with sexual dysfunction and 109 age-matched, normal volunteers. The mean IS scores were 10.6 and 5.5 for controls and patients, respectively, and the mean OS scores were 8.6 and 4.4 for controls and patients, respectively. Moreover, Patients on average scored 7.0 in sexual desire domain and patients scored 6.3. For the making of FSFI [8], the test was administered to women suffering from Female Sexual Arousal Disorder (FSAD) and healthy controls. Mean scores for the satisfaction domain was 8.2 and 12.8 for FSAD and controls, respectively, and mean sexual desire domain score were 4.7 and 6.9 for FSAD and controls, respectively. Therefore, we believe that our chosen cut-off can correctly discriminate between those with normal or low sexual desire and satisfaction.

Last, we did not include data on the impact of COVID19 pandemic on the sexual health of sexual and gender minorities such as lesbians, gay, bisexual and transgender people, and people living with HIV. 
To our knowledge, this is one of the few studies using validated questionnaires for both males and females to assess sexual well-being and psychometric alterations during COVID quarantine.

Our study shows a picture of sexual satisfaction and depressive symptoms in a young population during COVID lockdown. During this particular time, we found very low levels of sexual satisfaction throughout the responders. Health care workers showed a higher proportion of low sexual desire. A low sexual desire was associated with being female, being a health care worker, having children at home, living with the partner, and having low sexual satisfaction. The end of social restrictions and the return to normal daily life will be essential to restore people psychological and sexual well-being. Psychological and sexual counselling might be in people who will not be able to solve these problems the end of quarantine. Further analyses and a follow-up after the lockdown are required to analyse the real impact that this worldwide pandemic will have on our sexual life.

\section{Compliance with ethical standards}

Conflict of interest The authors declare that they have no conflict of interest.

Publisher's note Springer Nature remains neutral with regard to jurisdictional claims in published maps and institutional affiliations.

\section{References}

1. Report of clustering pneumonia of unknown etiology in Wuhan City. [Internet]. Wuhan Municipal Health Commission. 2019. http://wjw.wuhan.gov.cn/front/web/showDetail/2019123108989

2. Li Q, Guan X, Wu P, Wang X, Zhou L, Tong Y, et al. Early transmission dynamics in Wuhan, China, of Novel Coronavirus-infected pneumonia. N Engl J Med. 2020;382: 1199-207. https://www.nejm.org/doi/10.1056/NEJMoa2001316

3. Wu Z, McGoogan JM. Characteristics of and Important Lessons from the Coronavirus Disease 2019 (COVID-19) Outbreak in China: Summary of a Report of 72314 Cases from the Chinese Center for Disease Control and Prevention [Internet]. Vol. 323, JAMA - J Am Med Assoc. 2020. p. 1239-42. https://jamanetwork.com/

4. Sanford A. Coronavirus: Half of humanity now on lockdown as 90 countries call for confinement I Euronews. Euronews [Internet]. 2020 [cited 2020 Aug 10]; https://www.euronews.com/2020/04/ 02/coronavirus-in-europe-spain-s-death-toll-hits-10-000-afterrecord-950-new-deaths-in-24-hou

5. Cocci A, Presicce F, Russo GI, Cacciamani G, Cimino S, Minervini A. How sexual medicine is facing the outbreak of COVID-19: experience of Italian urological community and future perspectives [Internet]. International Journal of Impotence Research. Springer Nature; 2020 [cited 2020 Aug 10]. p. 1-3. https://doi.org/10.1038/s41443-020-0270-4

6. Angst J. Sexual problems in healthy and depressed persons. Int Clin Psychopharmacol. Lippincott Williams and Wilkins; 1998 [cited 2020 Aug 9];13 Suppl 6. https://pubmed.ncbi.nlm.nih.gov/9728667/
7. Rosen RC, Riley A, Wagner G, Osterloh IH, Kirkpatrick J, Mishra A. The international index of erectile function (IIEF): a multidimensional scale for assessment of erectile dysfunction. Urology. 1997;49:822-30. http://www.ncbi.nlm.nih.gov/pubmed/9187685

8. Rosen R, Brown C, Heiman J, Leiblum S, Meston C, Shabsigh R, et al. The female sexual function index (Fsfi): a multidimensional self-report instrument for the assessment of female sexual function. J Sex Marital Ther. 2000;26:191-205. https://pubmed.ncbi. nlm.nih.gov/10782451/

9. Filocamo MT, Serati M, Li Marzi V, Costantini E, Milanesi M, Pietropaolo A, et al. The female sexual function index (FSFI): linguistic validation of the italian version. J Sex Med. 2014;11:447-53. https://pubmed.ncbi.nlm.nih.gov/24224761/

10. Beck AT, Steer RA, Carbin MG. Psychometric properties of the Beck depression inventory: twenty-five years of evaluation. Clin Psychol Rev [Internet] Pergamon. 1988;8:77-100. https://www. sciencedirect.com/science/article/pii/0272735888900505

11. Bottesi G, Ghisi M, Altoè G, Conforti E, Melli G, Sica C. The Italian version of the Depression Anxiety Stress Scales-21: Factor structure and psychometric properties on community and clinical samples. 2015; https://doi.org/10.1016/j.comppsych.2015.04.005

12. Beck AT, Steer RA, Brown GK. Manual for the Beck depression inventory-II. San Antonio, TX: Psychol Corp; 1996.

13. Schiavi MC, Spina V, Zullo MA, Colagiovanni V, Luffarelli P, Rago R. et al. Love in the time of COVID-19: sexual function and quality of life analysis during the social distancing measures in a group of Italian reproductive-age women. J Sex Med. 2020;17:1407-13. /pmc/articles/PMC7342024/?report=abstract.

14. Li G, Tang D, Bin S, Wang C, Shen Q, Xu C, et al. Impact of the COVID-19 pandemic on partner relationships, sexual and reproductive health: a cross-sectional online survey (Preprint). J Med Internet Res. 2020.

15. Cocci A, Giunti D, Tonioni C, Cacciamani G, Tellini R, Polloni $\mathrm{G}$, et al. Love at the time of the Covid-19 pandemic: preliminary results of an online survey conducted during the quarantine in Italy. Int J Impot Res. 2002;32:556-7.

16. Yuksel B, Ozgor F. Effect of the COVID-19 pandemic on female sexual behavior. Int J Gynecol Obstet. 2020;150:98-102. https:// onlinelibrary.wiley.com/doi/abs/10.1002/ijgo.13193

17. Schreiner-Engel P, Schiavi RC. Lifetime psychopathology in individuals with low sexual desire. J Nerv Ment Dis. 1986;174:646-51. https://pubmed.ncbi.nlm.nih.gov/3772353/

18. Araujo AB, Durante R, Feldman HA, Goldstein I, Mckinlay JB. The relationship between depressive symptoms and male erectile dysfunction: cross-sectional results from the Massachusetts male aging study. Psychosom Med. 1998;60:458-65. https://pubmed. ncbi.nlm.nih.gov/9710291/

19. Feldman HA, Goldstein I, Hatzichristou DG, Krane RJ, McKinlay JB. Impotence and its medical and psychosocial correlates: results of the Massachusetts male aging study. J Urol 1994;151:54-61. https://pubmed.ncbi.nlm.nih.gov/8254833/

20. Strand J, Wise TN, Fagan PJ, Schmidt, Chester W. Erectile dysfunction and depression: category or dimension? J Sex Marital Ther. 2002;28:175-81. https://jhu.pure.elsevier.com/en/publica tions/erectile-dysfunction-and-depression-category-or-dimension-4

21. Corona G, Ricca V, Bandini E, Mannucci E, Petrone L, Fisher $\mathrm{AD}$, et al. Association between psychiatric symptoms and erectile dysfunction. J Sex Med. 2008;5:458-68. https://linkinghub. elsevier.com/retrieve/pii/S1743609515319354

22. Dwulit AD, Rzymski P. The potential associations of pornography use with sexual dysfunctions: an integrative literature review of observational studies. J Clin Med. 2019;8:914. /pmc/articles/ PMC6679165/?report $=$ abstract 